\title{
NOTE
}

\section{An integrated circuit for stepwise or continuous microdrive advancement}

\section{MATTHEW A. LAITINEN, MICHAEL L. WOODRUFF, and RONALD H. BAISDEN \\ College of Medicine, East Tennessee State University, Johnson City, Tennessee 37614}

A circuit for inexpensive automation of a hydraulic microdrive is described. The circuit uses two integrated timing circuits with output periods defined by resistancecapacitance circuits. The output of this circuit operates a relay that controls a small electric motor that runs the microdrive. This system can operate in either a continuous mode for long-term infusion of solutions or in a stepwise fashion for lowering electrodes. When attached to an appropriate microdrive, the circuit can be employed in experiments in which brain-behavior relationships are investigated within anatomical, pharmacological, or electrophysiological paradigms.

Microdrive units have many applications in research directed toward elucidation of the relationship between various physiological parameters and behavior. Such units can be used to lower electrodes for recording various types of behavior-related neuronal potentials or, when attached to appropriate in-dwelling cannulas, for infusion of substances into either the brain or the circulatory system. Some units (e.g., Trent-Wells Model 3-0590) are not constructed with the necessary motor and control circuitry for automated and timed operation. Other units have the circuitry and motor for automated advancement but are comparatively expensive (e.g., Kopf Model 607WCP, 1980 price: \$4,235; Stoelting Company Standard Model 51422, 1980 price: $\$ 3,475$ ). The system described in this note provides a low-cost electronic circuit that drives an inexpensive motor geared to a Trent-Wells Model 3-0590 hydraulic microdrive.

Timing for the operation of the microdrive is controlled by two integrated circuits (ICs) independently controlled from external resistance-capacitance (RC) networks. The timing generated by these ICs individually can be varied from microseconds to minutes with great accuracy. Temperature stability is $.005 \%$ per degree centigrade. For the combined circuit shown in Figure 1, the timing is continuously variable from 2 to $20 \mathrm{sec}$. The timing circuits control the rate of the hydraulicmicrodrive driving motor by means of a relay.

The circuit operates in the following manner. When Switch S1 is in the PULSE position, stepwise operation of the microdrive is produced by Timing Circuits IC1 and IC2, which determine the off and on periods of Relay K1 (see Figure 1). IC 1 and IC2 are connected to each other as a dual astable multivibrator. The on-off sequence is initiated by depressing Pushbutton Switch S2. Momentary closing of $\mathrm{S} 2$ produces a negative-going pulse that triggers IC2 Input Pin 2. This causes IC2 Output Pin 3 to change from low to high for a time period defined by the values of R4, R5, and $\mathrm{C} 4$. At the end of this time period, the IC2 output goes from high to low and triggers IC1 Input 2. IC1 Output Pin 3 will then go from low to high for a predetermined time, which is defined by $\mathrm{R} 1, \mathrm{R} 2$, and $\mathrm{C} 1$. When the output time for ICl is completed, the output goes to low and starts IC2. The cycle will repeat until stopped by the user. Timing Circuit IC2 determines the on time of Relay $\mathrm{K} 1$, and $\mathrm{IC} 1$ determines the off time. The LED indicator light $D 1$ is on whenever the relay $K 1$ is operated. Continuous operation of Relay K1, and therefore, the microdrive motor, can be produced by turning Switch S1 to the CONT position.

The power for the circuit is derived from a $+12-\mathrm{V} \mathrm{dc}$ regulated power supply that is operated from $110 \mathrm{~V} \mathrm{ac}$ introduced through Transformer $\mathrm{T} 1$. The $12 \cdot \mathrm{V}$ ac secondary output is rectified by a full-wave rectifier D3-D6 and filtered by Capacitor C9. An unregulated direct current of approximately $18 \mathrm{~V}$ is produced and fed into the $+12-\mathrm{V}$ dc regulator IC3.

All of the components depicted in Figure 1 are readily available from electronics suppliers and are very inexpensive. The total cost of the circuit was less than $\$ 50$ (1980 prices). A list of the necessary components is given in Table 1. Output of the circuit serves to regulate current flow to an electric motor that is geared to a Trent-Wells hydraulic microdrive. The circuit is

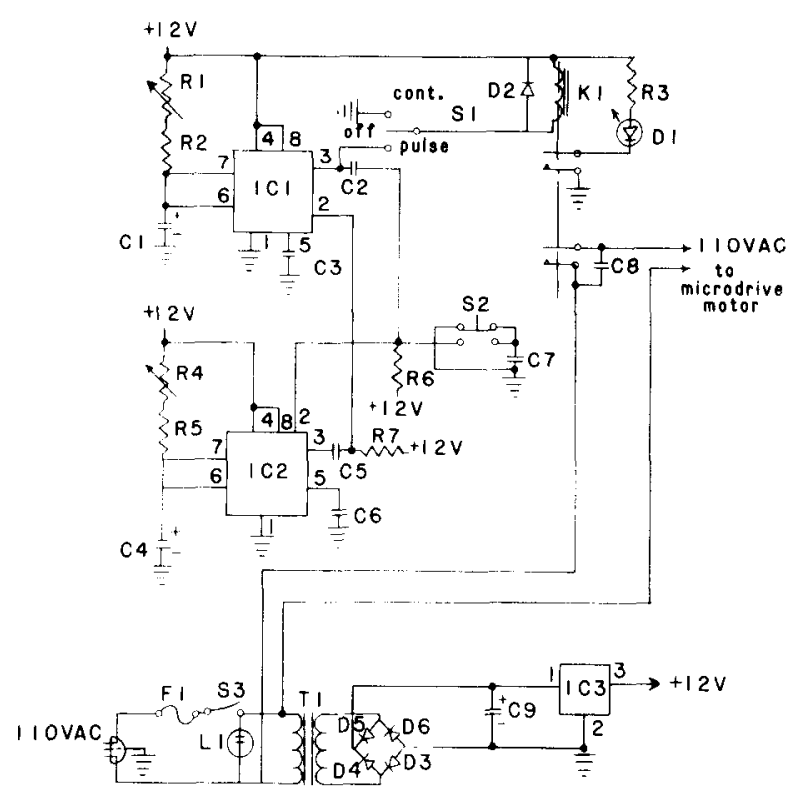

Figure 1. Circuit for control of microdrive motor. 
Table 1

A List of Components

$\mathrm{C} 1, \mathrm{C} 4$ Capacitor 10 microfarad $25 \mathrm{~V} \mathrm{dc}$

C2, C5 Capacitor .0012 microfarad

C3, C6 Capacitor .01 microfarad

C7 Capacitor .1 microfarad

C8 Capacitor 600 picofarad

C9 Capacitor 250 microfarad $50 \mathrm{~V} \mathrm{dc}$

D1 Diode LED

D2-D6 Diode IN4759

F1 Fuse AGC . 5 A

IC1, IC2 Timing Circuit MC1455

IC3 Voltage Regulator +12 V MC7812

K1 Relay 12 V dc R-10-E2-Y4-V185 (Potter and Brumfield)

L1 Lamp Assembly $120 \mathrm{~V}$ ac

R1, R4 Potentiometer 1 megohm Linear

R2, R5 Resistor $100 \mathrm{kohm} \pm 5 \% .25 \mathrm{~W}$

R6, R7 Resistor $27 \mathrm{kohm} \pm .25 \mathrm{~W}$

R3 Resistor $470 \mathrm{ohm} \pm .25 \mathrm{~W}$

S1 Switch SPDT Center Off

S2 Switch PBDT

S3 Switch SPST

T1 Transformer-Calectro D1-746 $12 \mathrm{~V}$ ac .5 A

enclosed, and the microdrive is mounted on Plexiglas. The motor we use turns at $1 \mathrm{rpm}$ and is manufactured by the Cramer Division of the Conrac Corporation. The motor is Type 117 and runs on $115 \mathrm{~V}$ ac. Similar motors with rpm values from .0004 to 20 are also available from Razel Scientific Instruments, P.O. Box 4054, Stamford, Connecticut 06907. The motors cost approximately $\$ 5$ each. The 1980 price of the Trent-Wells 3-0590 standard hydraulic microdrive is $\$ 855$. Inclusion of this commercial microdrive brings the entire unit cost to about $\$ 950$, but the system can perform all of the operations of devices costing from $\$ 3,000$ to $\$ 4,200$. This system has been used for intracranial injection of less than a microliter of horseradish peroxidase or 6hydroxydopamine (Woodruff \& Baisden, 1980) through a Hamilton microsyringe. It can also be used for intravenous infusion of much larger volumes.

\section{REFERENCE}

WoodrufF, M. L., \& Baisden, R. H. Increased catecholamine fluorescence in the anterior commissure of the rat induced by intraseptal 6-hydroxydopamine. Anatomical Record, 1980, 196, 255A.

(Received for publication August 6, 1980; accepted August 8, 1980.) 